\title{
Older psychiatric in-patients' knowledge about psychotropic and non-psychotropic medications
}

\author{
Sri Perecherla, ${ }^{1}$ Alastair J. D. Macdonald ${ }^{2}$
}

The Psychiatrist (2011), 35, 220-224, doi: 10.1192/pb.bp.109.026500

${ }^{1}$ Croydon University Hospital ${ }^{2}$ Institute of Psychiatry, London Correspondence to Sri Perecherla (s.perecherla@nhs.net)

First received 6 May 2009, final revision 29 Oct 2010, accepted 7 Dec 2010

\begin{abstract}
Aims and method We hypothesised that, because of stigma associated with mental illness, patients' knowledge of psychotropic medication would be less than that of non-psychotropic medication. We set out to establish the extent of knowledge about these medications using a cross-sectional survey of in-patients over 65 years of age in south London.
\end{abstract}

Results Of the study population $(n=86), 42 \%$ demonstrated an understanding of the purpose of taking both psychotropic and non-psychotropic medication, 15\% understood only their psychotropic medication, $16 \%$ understood only their nonpsychotropic medication and $27 \%$ understood neither medication. A surprising finding was that more than $20 \%$ of these patients with cognitive impairment were not able to recount their legal status; this factor was independently related to whether or not they knew the purpose of their psychotropic medication, and also whether or not they knew the purpose of neither type of medication.

Clinical implications Poor knowledge of medication has been associated with nonadherence and relapse. Older adults are likely to receive multiple medications because of their increased susceptibility to physical and psychiatric disorders with increasing age, so even more emphasis has to be placed on increasing knowledge of medication.

Declaration of interest None.
Pharmacological treatment is considered the mainstay of the management of serious mental illness. Over the course of a year, about three-quarters of people prescribed psychotropic medication will discontinue their medication, often coming to the decision themselves and without informing a health professional. ${ }^{1}$ Cooper et al found that two-fifths of their study participants taking psychotropic medication reported discontinuing medication because they did not want it or did not think it necessary. ${ }^{2}$ Treatment non-adherence has been consistently associated not only with relapse and rehospitalisation ${ }^{3}$ but also with higher costs, ${ }^{4}$ and it remains one of the greatest challenges in psychiatry. $^{5}$

Although older adults constitute approximately a fifth of the population in England, ${ }^{6}$ they receive about half of all prescriptions. ${ }^{7}$ Older adults often take a variety of drugs for several conditions, ${ }^{8}$ and non-adherence is reported to have been a problem in as many as $50 \%$ of them. ${ }^{9}$ Lack of information about medication has been associated with nonadherence in older patients, ${ }^{10}$ and education can significantly improve medication knowledge and adherence. ${ }^{11}$

There is little published research on the knowledge patients have of their medications, ${ }^{12}$ and almost all studies appear to be of psychiatric or medical patients under the age of 65 years. We hypothesised that older psychiatric in-patients would have a low level of medication knowledge. We also hypothesised that, owing to the stigma associated with mental illness, their knowledge of psychotropic medication would be comparatively less than that of non-psychotropic medication. This study aimed to compare knowledge of psychotropic and non-psychotropic medication in this group of patients.

\section{Method}

The study was carried out within all six older adult mental health in-patient units in a large urban and suburban mental health trust. Visits were made by one of us (S.P.) to the units between April 2006 and May 2007. All in-patients were considered for inclusion. Inclusion criteria were as follows:

- aged 65 years or older;

- in-patient on psychiatric unit for older adults;

- receiving at least one regular psychotropic and nonpsychotropic medication;

- able to communicate in English;

- well enough to be able to sit through the interview;

- able to demonstrate capacity to consent to participate in the study;

- Mini-Mental State Examination (MMSE) ${ }^{13}$ score of 24 or above. 
Those people who possibly met the inclusion criteria were identified with the assistance of nursing staff from the list of in-patients on the units. Potential participants were approached, the purpose of the study was explained, and an information sheet was provided, before obtaining written consent. Patients who were unable to demonstrate a clear understanding of their involvement in the study were considered to lack capacity to consent to the study and were excluded. An MMSE was carried out for the potential participants if there was no documented evidence of it being carried out in the 3 months before the day of the study interview. Only people with an MMSE score of 24 or above were included in the study, in order to try to reduce the impact of cognitive impairment as a cause of poor knowledge of medication.

Demographic, diagnostic and medication data on participants who provided written consent were collected from their in-patient medical records. It was not possible to ascertain the duration of treatment in most participants as the date of commencement of medication was not clearly documented. If a participant was prescribed more than one psychotropic, then we ascertained the primary psychiatric diagnosis and selected the longest-term treatment option for it. For example, if bipolar disorder was the primary diagnosis, then a mood stabiliser rather than an antipsychotic drug would be selected. In the non-psychotropic category, medications prescribed for systemic diseases were selected in preference to analgesics and supplements. Only medications prescribed to be taken on a regular basis were selected in preference to others.

One of us (S.P.) then conducted a semi-structured interview in which the participant was asked to list the names of all the medications they were receiving regularly. If the participant recalled the name of the drug selected by the investigator, then the investigator proceeded to ask the remaining questions. If the participant failed to recall the name of the drug, then the investigator stated the name of the selected drug before proceeding to ask the remaining questions. The participant's level of knowledge of the medication was assessed by the knowledge of:

- the name of the medication;

- the dose in grams, milligrams or micrograms;

- the frequency of use;

- the name of the condition for which the medication was prescribed and the reason for prescribing the drug (approximations were accepted, e.g. 'to stop hearing voices' or 'to control blood pressure');

- the duration for which the prescribed drug was advised to be taken;

- knowledge of side-effects;

- the action to be taken if a dose is missed;

- the addictive properties, if any, of the medication;

- the effect of the medication on the participant's ability to drive or operate machinery;

- the effects of alcohol consumption on the medication;

- the interactions, if any, of the medication;

- the possible consequences of non-adherence to the medication.

In addition, questions were asked about the perceived usefulness of the medication and the person's willingness to adhere to the medication. We decided the single question that would best capture the participant's basic knowledge of any medication was: 'Do you know the purpose of taking your medication?' In this article we focus on the participants' response to this question.

Our primary null hypothesis was: 'When psychiatric in-patients over the age of 65 are asked the question "Do you know the purpose of taking this medication?", the difference between the proportions giving a "don't know" response about psychotropic, as opposed to nonpsychotropic, medication is less than $30 \%$.' For example, if $20 \%$ understood the purpose of their psychotropic medication and $45 \%$ their non-psychotropic medication, then the difference (25\%) would allow the null hypothesis (no important difference) to stand. A sample size of 86 was required to demonstrate such a difference $(\alpha 0.05, \beta 0.2)$.

Statistical analyses were carried out using SPSS for Windows, version 15. The null hypothesis was tested using confidence intervals for proportions. Primary analysis was carried out to identify the proportions of participants who demonstrated knowledge of the purpose of taking psychotropic medication, non-psychotropic medication, both medications and neither medication. Secondary analysis was carried out to determine whether knowledge of the purpose of taking the medication was associated with individual variables such as gender and age. Those variables that were found by univariate analysis to be associated with knowledge were subjected to a multivariate logistic regression analysis with block entry to determine whether or not the association of each variable was independent of other variables. For this purpose, age was divided by 10 to aid interpretation, and diagnosis was condensed into two groups: participants in ICD-10 ${ }^{14}$ chapters F30, F40 or F50 (broadly, mood and anxiety disorders) and the remainder. Finally, we explored whether knowledge of the medication was related to recall of having received information and a desire for further information.

The study was approved by the Joint South London and Maudsley and the Institute of Psychiatry NHS Research Ethics Committee.

\section{Results}

A total of 92 patients were approached in order to identify 86 participants regularly taking at least one psychotropic medication and one non-psychotropic medication. Five participants did not consent and one participant scored below 24 on the MMSE. None of the patients approached lacked capacity to consent to the study.

Sixty of the study sample $(69.9 \%)$ were female. The mean age was 76.6 years $($ s.d. $=6.0)$ for females and 74.3 years (s.d. =3.7) for males. The mean length of formal education was 10.4 years $($ s.d. $=2.6)$. Sixty-two of the patients $(72.1 \%)$ did not have a partner. Seventy-nine of the patients $(91.9 \%)$ lived in their home (own or rented). Twenty-three of the patients (26.7\%) did not know or incorrectly reported their legal status. Of the $72(83.7 \%)$ informal patients, 18 (25\%) did not know their legal status; of the 14 (16.3\%) legally detained patients, 5 (35.7\%) did not know their legal status. An ICD-10 diagnosis of depressive disorders was made in 41 patients (48\%), bipolar disorder in 


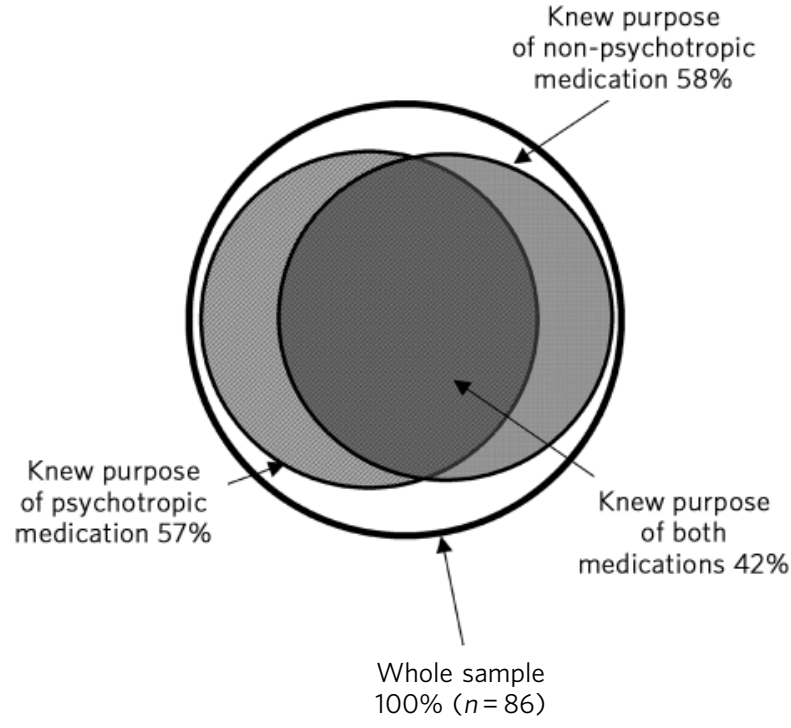

Fig 1 Scaled Venn diagram showing knowledge of purpose of psychotropic and non-psychotropic medication in 86 older psychiatric in-patients. The areas within each circle and arc are proportional to the numbers represented. ${ }^{15}$

14 patients (16\%), schizophrenia in 8 patients (9\%), schizoaffective disorder in 6 patients $(7 \%)$, other psychotic disorders in 7 patients (8\%), and other disorders (e.g. anxiety disorder) in 10 patients (12\%). The key psychotropic drug used was an antidepressant in 48 patients (55.8\%) and an antipsychotic or a mood stabiliser in the remainder. The key non-psychotropic drug used was for cardiovascular disorder in 51 patients $(59.3 \%)$ and for other medical disorders in the remainder.

Of the 86 participants, $14 \%$ understood only the purpose of their psychotropic medication, $16 \%$ only the purpose of their non-psychotropic medication, $43 \%$ the purpose of both their psychotropic and non-psychotropic medication, and $27 \%$ the purpose of neither medication. Fig. 1 shows a scaled Venn diagram in which the areas bounded by the lines are proportional to the numbers represented. Overall, the proportion of participants who did not know the purpose of their psychotropic medication was $43.0 \%$ (95\% CI 32.3-53.7), while the proportion who did not know the purpose of their non-psychotropic medication was 40.7\% (95\% CI 30.1-51.3). The null hypothesis was thus not disproved.

Table 1 shows the relationship between knowledge of medication and various factors. Of the 86 participants, 49 (57\%) were able to demonstrate an understanding of the purpose of their psychotropic medication. Six variables (female gender, living in own household, mood/anxiety diagnosis, informal status, knowledge of legal status, taking antidepressant as key medication) were shown by univariate analysis to have an association with participants' knowledge of their psychotropic medication, but only two of the six variables (mood/anxiety diagnosis and knowledge of legal status) were shown by multivariate logistic regression to have an independent association with this knowledge. Patients with psychiatric diagnosis listed within ICD-10 chapters F30-59 (broadly, mood and anxiety disorders) were nearly seven times more likely to know the purpose of their psychotropic medication than those with other diagnoses, independent of the other five variables included above $(\mathrm{OR}=6.90,95 \%$ CI 1.72-27.62, $P=0.006)$. Patients who knew their legal status were over four times more likely to know the purpose of their psychotropic medication than those who did not, independent of the other five variables included above (OR=4.63, 95\% CI 1.23-17.33, $P=0.023)$.

Fifty-one participants (59\%) were able to demonstrate an understanding of the purpose of their non-psychotropic medication. Two variables (lower age, knowledge of legal status) were shown by univariate analysis to have an association with participants' knowledge of their nonpsychotropic medication, and both of these variables were shown by multivariate logistic regression to have an independent association with this knowledge. Patients in each 10-year age cohort were over twice as likely to know the purpose of their non-psychotropic medication than the next older cohort, independent of their knowledge of their legal status (OR $=2.62,95 \%$ CI 1.07-6.41, $P=0.035)$. Patients who knew their legal status were nearly three times more likely to know the purpose of their non-psychotropic

\begin{tabular}{|c|c|c|c|c|c|c|c|c|}
\hline \multirow[b]{2}{*}{ Variable } & \multicolumn{2}{|c|}{$\begin{array}{c}\text { Knowledge of } \\
\text { psychotropic medication }\end{array}$} & \multicolumn{2}{|c|}{$\begin{array}{l}\text { Knowledge of non- } \\
\text { psychotropic medication }\end{array}$} & \multicolumn{2}{|c|}{$\begin{array}{l}\text { Knowledge of both } \\
\text { medications }\end{array}$} & \multicolumn{2}{|c|}{$\begin{array}{l}\text { Knowledge of neither } \\
\text { medication }\end{array}$} \\
\hline & $\begin{array}{l}\text { Present } \\
(n=49)\end{array}$ & $\begin{array}{l}\text { Absent } \\
(n=37)\end{array}$ & $\begin{array}{l}\text { Present } \\
(n=51)\end{array}$ & $\begin{array}{l}\text { Absent } \\
(n=35)\end{array}$ & $\begin{array}{l}\text { Present } \\
(n=37)\end{array}$ & $\begin{array}{l}\text { Absent } \\
(n=49)\end{array}$ & $\begin{array}{l}\text { Present } \\
(n=23)\end{array}$ & $\begin{array}{l}\text { Absent } \\
(n=63)\end{array}$ \\
\hline Female, \% & 81.6 & 54.1 & 76.4 & 60 & 83.8 & 59.2 & 52.2 & 76.2 \\
\hline Not living in care home, \% & 98 & 83.8 & 94.1 & 88.6 & 100 & 85.7 & 87 & 93.7 \\
\hline $\begin{array}{l}\text { Diagnosis in ICD-10 codes } \\
\text { F30-59, \% }\end{array}$ & 89.8 & 45.9 & 66.7 & 77.1 & 86.5 & 59.2 & 65.2 & 73 \\
\hline Legal status 'informal', \% & 93.9 & 70.3 & 82.4 & 85.7 & 91.9 & 77.6 & 78.3 & 85.7 \\
\hline Knows legal status, \% & 85.7 & 56.8 & 82.4 & 60 & 89.2 & 61.2 & 52.2 & 81 \\
\hline Taking antidepressant drug, \% & 73.5 & 32.4 & 52.9 & 60 & 70.3 & 44.9 & 47.8 & 58.7 \\
\hline Taking non-cardiac drug, \% & - & - & - & - & 35.1 & 44.9 & 43.5 & 39.7 \\
\hline Mean age, years & 75.8 & 76 & 74.7 & 77.5 & 74.5 & 76.9 & 76.4 & 75.7 \\
\hline
\end{tabular}


medication than those who did not, independent of their age $(\mathrm{OR}=2.93,95 \%$ CI $1.06-8.14, P=0.038)$.

Thirty-seven participants (43\%) were able to demonstrate an understanding of the purpose of taking both psychotropic and non-psychotropic medication. Six variables (female gender, lower age, living in own household, mood/anxiety diagnosis, knowledge of legal status, taking antidepressant as key psychotropic medication) were shown by univariate analysis to have an association with participants' knowledge, but only three of the six variables (female gender, lower age, mood/anxiety diagnosis) were shown by multivariate logistic regression to have an independent association with this knowledge: female gender $(\mathrm{OR}=3.71,95 \%$ CI 1.08-12.68, $P=0.037)$, lower age $(\mathrm{OR}=3.91,95 \%$ CI $1.27-12.0, P=0.017)$ and a psychiatric diagnosis listed within ICD-10 chapters F30-59 (OR $=4.14$, 95\% CI 1.02-16.74, $P=0.046$ ).

Twenty-three participants (26\%) were unable to demonstrate an understanding of purpose of taking either their psychotropic or their non-psychotropic medication. Two variables (male gender, lack of knowledge of legal status) were shown by univariate analysis to have an association with participants' lack of knowledge, but only one variable (lack of knowledge of legal status) was shown by multivariate logistic regression to have an independent association with participants' lack of knowledge about either medication $(\mathrm{OR}=3.45,95 \%$ CI $1.20-9.96, P=0.022)$.

Of the 49 patients $(34.7 \%)$ who knew the purpose of their psychotropic medication, 17 did not recall having received any information on this, as opposed to 27 of the 37 patients $(73.0 \%)$ who did not know the purpose of their psychotropic medication $\left(\chi^{2}=12.4, \quad\right.$ d.f. $\left.=1, \quad P<0.001\right)$. Overall, 45 patients (52.3\%) said they wanted more information about their psychotropic medication; 20 $(40.8 \%)$ of those who knew the purpose of their psychotropic medication and 25 (67.6\%) of those who did not know the purpose of their psychotropic medication.

Of the 51 patients (13.7\%) who knew the purpose of their non-psychotropic medication, 7 did not recall having received any information, compared with 30 of the 35 patients (85.7\%) who did not know the purpose of their non-psychotropic medication $\left(\chi^{2}=43.8\right.$, d.f. $\left.=1, P<0.001\right)$. Overall, 40 patients $(46.5 \%)$ said they wanted more information about their non-psychotropic medication; 18 $(35.3 \%)$ of those who knew the purpose of their nonpsychotropic medication and $22(62.9 \%)$ of those who did not know the purpose of their non-psychotropic medication.

\section{Discussion}

Our study was limited to in-patients from a defined geographical area and the sample size was modest. We deliberately selected patients in whom nurses perceived little or no cognitive impairment, but the possibility that impairment was responsible for some of the misunderstanding about medication was not eliminated. The criteria on which patients' knowledge of the purpose of their medication was assessed were somewhat subjective, as was the choice by the investigator of medication about which to enquire. Patients' knowledge was not assessed masked to the variables used in the secondary analysis. We were not able to ascertain the length of treatment, which potentially could be associated with knowledge of medication.

The main finding was that less than half of a group of relatively cognitively intact older in-patients understood the purpose of both one key psychotropic medication and one key non-psychotropic medication that they were taking. This was in keeping with studies of knowledge in younger psychiatric patients. ${ }^{12}$ Male gender and older age were associated with this lack of knowledge. ${ }^{9}$ Lack of information about medication has been associated with non-adherence in older patients. ${ }^{10}$ We found a strong association between recall of receiving information about medication and knowledge of its purpose, but this could be related to general recall problems in this patient group and may not reflect staff efforts to give information. In the trust where this study was conducted, pharmacies supply manufacturers' patient information leaflets with dispensed medication. However, pharmacists assume that it is the key worker's responsibility to ensure that the patient has access to information about their medication. We noted that there was a variation in the provision of leaflets routinely for all prescriptions on in-patient wards during the time of this study.

No patient approached lacked capacity to consent to the study, and yet $26.7 \%$ of patients did not know their legal status, illustrating the contextual nature of capacity assessments; capacity for one decision does not guarantee capacity for another. Some of these older informal patients believed that being admitted to a psychiatric ward without any application of legislation was impossible. Knowledge of legal status was independently related to whether or not the patient knew the purpose of their psychotropic medication, and also whether or not they knew the purpose of neither type of medication. This implies that lack of knowledge of the purpose of medication is part of a more general lack of knowledge about their condition and treatment rather than a specific scotoma, even in relatively cognitively intact patients. This finding suggests the need for further research in this area.

We found no evidence that patients discriminated between psychotropic and non-psychotropic treatment; if stigma against mental illness affects patients' knowledge of medication, then this was not reflected in this study. These data suggest that more work needs to be done in improving patients' understanding of their condition, treatment and legal status. The Mental Capacity Act was not in force at the time of this study, but the study raises concerns that lack of knowledge of the purpose of medication may impair capacity to consent to taking medication, and this may require further study.

\section{Acknowledgements}

Thanks to Dr James Warner, Jonathon Artingstall and Bob Blizard for their advice and support.

\section{About the authors}

Sri Perecherla is Consultant Psychiatrist at Woodcote House, Croydon University Hospital. Alastair J. D. Macdonald is Visiting Professor of 
Psychiatry in the Health Services and Population Research Department, Institute of Psychiatry, London.

\section{References}

1 Mitchell AJ, Selmes T. Why don't patients take their medicine? Reasons and solutions in psychiatry. Adv Psychiatr Treat 2007; 13: 336-46.

2 Cooper C, Bebbington $P$, King M, Brugha T, Meltzer $H$, Bhugra $D$, et al. Why people do not take their psychotropic drugs as prescribed: results of the 2000 National Psychiatric Morbidity Survey. Acta Psychiatr Scand 2007; 116: 47-53.

3 Pratt SI, Mueser KT, Driscoll M, Wolfe R, Bartels SJ. Medication nonadherence in older people with serious mental illness: prevalence and correlates. Psychiatr Rehabil J 2006; 29: 299-310.

4 Knapp M, King D, Pugner K, Lapuerta P. Non-adherence to antipsychotic medication regimens: associations with resource use and costs. $\mathrm{Br}$ Psychiatry 2004; 184: 509-16.

5 Nosé M, Barbui C, Gray R, Tansella M. Clinical interventions for treatment non-adherence in psychosis: meta-analysis. $\mathrm{Br} J$ Psychiatry 2003; 183: 197-206

6 Department of Health. National Service Framework for Older People. Department of Health, 2001.
7 Department of Health. Prescriptions Dispensed in the Community: Statistics for 1989-99 - England. Statistical Bulletin, 2000.

8 National Prescribing Centre. Prescribing for the older person. MeReC Bulletin 2000; 11: 37-40

9 Marinker M. From Compliance to Concordance: Achieving Shared Goals in Medicine Taking. Royal Pharmaceutical Society of Great Britain, 1997.

10 Wetherell $\mathrm{JL}$, Unutzer J. Adherence to treatment for geriatric depression and anxiety. CNS Spectr 2003; 8 (12 suppl 3): 48-59.

11 Lowe CJ, Raynor DK, Courtney EA, Purvis J, Teale C. Effects of self medication programme on knowledge of drugs and compliance with treatment in elderly patients. BMJ 1995; 310: 1229-31.

12 Brown KW, Billcliff N, McCabe E. Informed consent to medication in long-term psychiatric in-patients. Psychiatr Bull 2001; 25: 132-4.

13 Folstein M, Folstein S, McHugh P. 'Mini-Mental State', a practical method for grading the cognitive state of patients for the clinician. J Psychiatr Res 1975; 12: 189-98.

14 World Health Organization. The ICD-10 Classification of Mental and Behavioural Disorders: Clinical Descriptions and Diagnostic Guidelines. WHO, 1992.

15 Chow S, Ruskey F. Drawing area-proportional Venn and Euler diagrams. In Graph Drawing (ed G Liotta): 466-77. Springer, 2004.

\title{
Stability of the diagnosis of first-episode drug-induced psychosis
}

\author{
Aravind Komuravelli, ${ }^{1}$ Rob Poole, ${ }^{2,3}$ Robert Higgo ${ }^{4}$
}

The Psychiatrist (2011), 35, 224-227, doi: 10.1192/pb.bp.110.032524

${ }^{1}$ Whiston Hospital, Prescott; ${ }^{2}$ Glyndwr University, Wrexham; ${ }^{3}$ Wrexham Academic Unit; ${ }^{4}$ Liverpool Assertive Outreach Team

Correspondence to Aravind Komuravelli

(aravind.komuravelli@5bp.nhs.uk)

First received 6 Sep 2010, final revision 24 Nov 2010, accepted 10 Dec 2010
Aims and method To assess the stability of the diagnosis of first-episode druginduced psychosis over a follow-up period of at least 2 years. Patients with no psychiatric history who had been discharged from in-patient care between January 2002 and April 2006 with a firm diagnosis of drug-induced psychosis were identified. Follow-up information for at least the next 2 years or until discharge from mental health services was collected retrospectively from psychiatric records.

Results Nearly all of the patients who remained under psychiatric follow-up had a change in diagnosis, most commonly to a schizophreniform disorder. Those who were retained in follow-up had significantly longer index admissions than those discharged to primary care $(P=0.05)$

Clinical implications This study suggests that many individuals diagnosed with drug-induced psychosis are further diagnosed as having a functional psychosis, usually schizophreniform in nature. This is compatible with the suggestion that individuals diagnosed with drug-induced psychosis are experiencing either the effects of drug intoxication or an ordinary functional psychosis complicated by incidental drug use.

Declaration of interest None.
There is continuing interest among researchers and policymakers in the relationship between cannabis use and schizophrenia. $^{1-3}$ This has revived interest in the nature of the conditions that attract the clinical diagnosis of druginduced psychosis. ${ }^{4}$ The concept of drug-induced psychosis has been criticised ${ }^{5}$ and it remains unclear whether the diagnosis reflects a valid diagnostic category or whether it is based on arbitrary assumptions regarding the causation of symptoms. A systematic review concluded that the inference of causation is not secure and that the condition 